\title{
Aplicação da Smart Contract nos Contratos de Gás Natural: Uma Análise Exploratória
}

The Smart Contract on Gas Trade: An Exploratory Analysis

Romulo Benites de Souza Luciano ${ }^{1}$

(iD) https://orcid.org/0000-0002-5375-6608

Instituto Brasileiro de Mercado de Capitais, Departamento de Pós-Graduação e Pesquisa em Administração, Rio de Janeiro, RJ, Brasil ${ }^{1}$ 


\title{
Resumo
}

Este artigo apresenta uma nova tecnologia de informação chamada Smart Contract (SC) como proposta para automação da gestão de contratos no processo de comercialização do gás natural. O centro decisor, ao avaliar essa opção, deve estimar com antecedência seu impacto para o futuro. A proposta de utilização da SC encontra fundamentos para implementação no contexto atual do mercado de gás natural brasileiro, englobando os principais players desse comércio, isto é, governo, organização e consumidor. Este artigo propõe uma abordagem exploratória sobre a SC, pois busca-se um melhor entendimento sobre seus atributos, haja vista que não há estudos anteriores com aplicação do SC ao peculiar mercado de gás brasileiro. A metodologia é qualitativa porque trata-se de aspectos subjetivos na utilização da tecnologia de informação. Foi com auxílio de questionário satisfatoriamente respondido por dois especialistas que contribuíram para o processo de compreensão da viabilidade do uso do SC. Apresento dois possíveis cenários na utilização da SC para fomentar a discussão considerando o status quo do mercado de gás brasileiro. A técnica de coleta de dados sobre os construtos foi feita através da pesquisa documental da emergente literatura sobre Smart Contract, Blockchain e o atual mercado de gás natural no Brasil.

Palavras-chave: Blockchain; smart contract; comercialização de gás; contratos.

\begin{abstract}
The article presents a new information technology named Smart Contract (SC) as a proposal for automating contract management in today's natural gas trade. The decision-maker should estimate in advance the impact of this technology for the future. The use of Smart Contract in the actual scenario of the Brazilian natural gas market involves some fundamental factors that encompass the main players of trade: Government, Organization, and Consumer. This article proposes an exploratory approach regarding Smart Contract to understand its attributes since no previous studies with SC have been conducted specifically for the Brazilian gas market. The qualitative methodology was selected because it analyses subjective aspects of the proposed information technology. Analysis included a questionnaire that was answered by two specialists who provided valuable insight into the feasibility of using the SC. I present two possible scenarios using Smart Contract to foster discussion considering the status quo of the Brazilian gas market. The data regarding the constructs was collected using documentary research of the emerging literature on Smart Contract, Blockchain, and the current gas market in Brazil.
\end{abstract}

Keywords: Blockchain; smart contract; gas trade; contracts.

JEL code: O14, K32, L14. 


\section{Introdução}

$\mathrm{O}$ atual mercado de gás natural brasileiro encontra desafios para o seu desenvolvimento na área de midstream e downstream (Ministério de Minas e Energia [MME], 2014 e Agência Nacional do Petróleo, Gás Natural e Biocombustíveis [ANP], 2011). Desde o monopólio na área de transporte de gás natural até a parte burocrática no negócio, conforme evidenciado na Figura . Vale lembrar que o contrato descrito na Figura 1 é de 2003 e que, atualmente, existem termos e condições gerais mais atuais no mercado de gás natural brasileiro. No entanto, ele serve como fonte básica para vislumbrar várias ações que podem ser automatizadas através do SC. Nesse sentido, para auxiliar os principais membros participantes nesse comércio, é apresentado a Smart Contract (SC), como uma tecnologia de informação capaz de automatizar a gestão do contrato. A fim de contribuir para a análise dessa nova tecnologia e conduzir o centro decisor ao julgamento foi utilizado um estudo de mapeamento sistemático sobre tópicos atuais de pesquisa em SC, conforme já utilizado com sucesso pelos autores Alharby e Moorsel (2017). Nesse artigo elaborado pelos autores, uma das lacunas identificadas para pesquisas futuras foi para que novas pesquisas considerassem o estudo de várias aplicações potenciais do SC. Portanto, este estudo visa contribuir de forma exploratória o tema aplicado ao mercado de gás brasileiro. A coleta de informações sobre os principais conceitos foi realizada na base de dados do ProQuest, Google Scholar, Elsevier, ScienceDirect e Emerald Insight. As palavras chave utilizadas na busca foram Blockchain, smart contract e contratos inteligentes, separados e em combinação, usando and e or, sem excluir os livros. Ainda, nas mesmas bases de dados citadas, a busca foi feita com os seguintes termos: contratos, gás natural, comércio de gás natural brasileiro, supply chain management, decision making process, contracts, energy, oil and gas e operations management separados e em combinação, usando and e or, sem excluir os livros.

O trabalho está organizado em seis seções, incluindo esta introdução. A próxima seção apresenta as características do atual mercado de gás natural brasileiro e a demonstração da quantidade de obrigações contratuais. A seção seguinte expõe as características da tecnologia Blockchain através da revisão da literatura emergente assim como foi feita na outra seção que apresenta as características da Smart Contract. A penúltima seção exibe a predição do uso do SC utilizada apara abordar o problema. Por fim, a última seção apresenta as conclusões. 


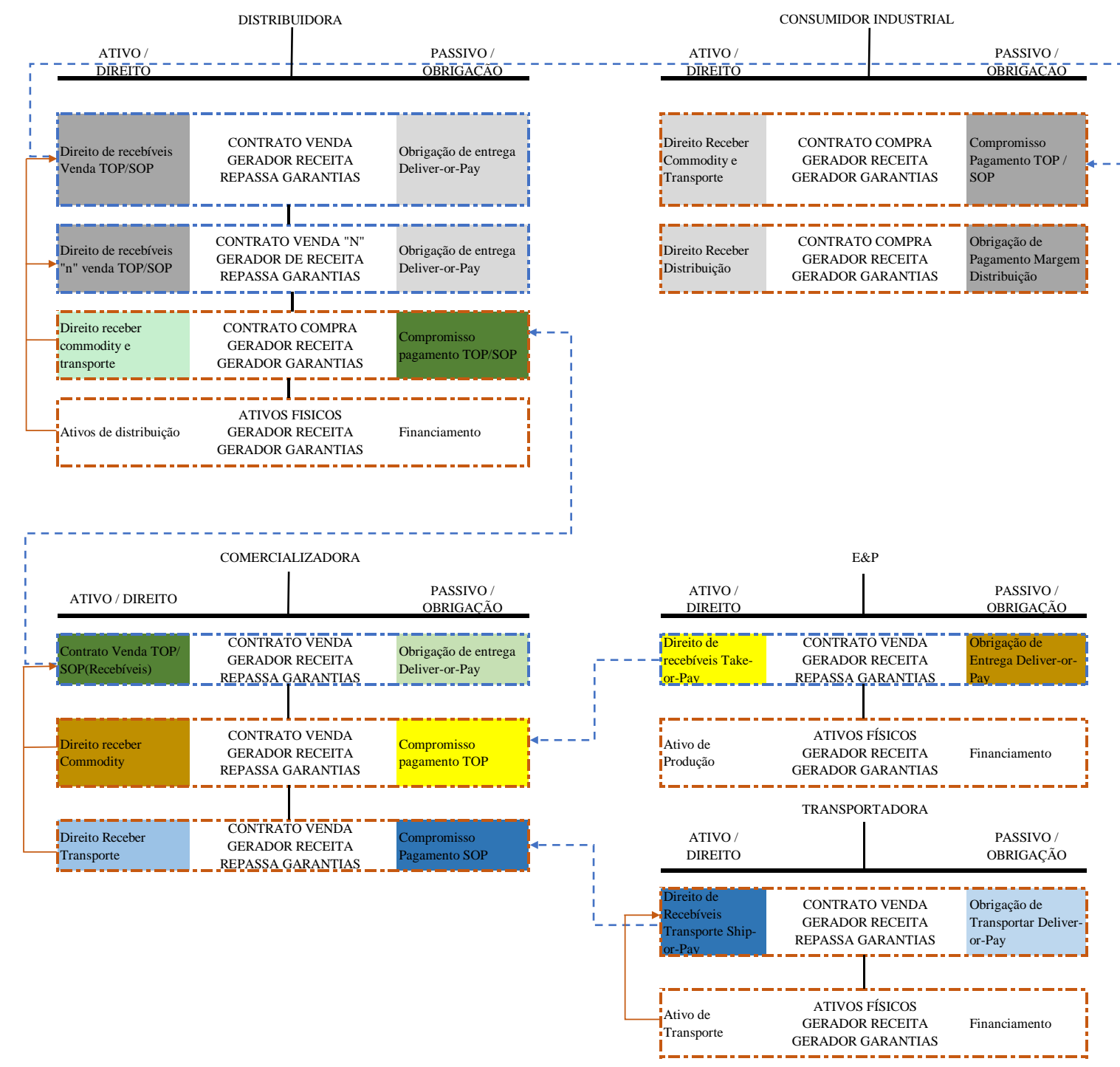

Indica transferência de garantias $----\rightarrow$

Indica a geração de receitas $\quad----\rightarrow$

Figura 1. Fluxograma dos Direitos e Obrigações Contratuais Utilizando a Analogia do Balanço Contábil de Ativos e Passivos

Fonte: Elaborado pelo autor a partir de Carvalhinho, J. C. L., Filho (2003). O valor da flexibilidade em cláusulas take-or-pay de contratos para fornecimento de gás natural industrial (pp. 120-138). (Dissertação de mestrado). Faculdade de Economia, Administração e Contabilidade, Universidade de São Paulo, São Paulo, SP, Brasil. Recuperado de http://www.teses.usp.br/teses/disponiveis/12/12139/tde-01102003-232151/en.php

\section{Comércio de Gás Natural no Brasil}

O Governo Federal Brasileiro promulgou a Lei $\mathrm{n}^{\circ}$ 9.478, de 6 de agosto de 1997 e conforme descrito, para incrementar, em bases econômicas, a utilização do gás natural e promover a livre concorrência, entre outros objetivos descritos na Lei.

Em seguida, com o propósito de melhorar a concorrência, foi publicada a Lei $n^{\circ} 11.909$, de 4 de março de 2009, mais conhecida como a Lei do Gás. As companhias internacionais observaram o mercado de gás brasileiro como promissor no longo prazo, visto que podem promover expansão da oferta de gás natural através de novas descobertas de jazidas e sua exploração (Gomes, 2014). Essa oferta poderá atender uma demanda de um mercado consumidor que ainda não é atendido pela atual 
infraestrutura. Demanda que poderá ser causada pelo fim do principal contrato de importação de gás natural da Bolívia em 2019 e a maior participação do gás natural na matriz energética do país (MME, 2014).

Especificamente sobre o gás natural, a Petrobras ainda é monopolista no fornecimento, pois é proprietária de grande parte da malha de gasodutos para o transporte, detém participação societária em quase todas as companhias de distribuição e governa o fluxo de gás entre os campos produtores e mercado consumidor (ANP, 2011).

Conforme explicitado na Confederação Nacional da Indústria (CNI, 2016), todos os produtores de gás vendem o seu produto para a Petrobras. A empresa compra o gás dos outros produtores e encarrega-se do tratamento, transporte e comercialização do gás para as distribuidoras, conforme Figura 2. A Petrobras é também responsável por toda a importação de gás realizada atualmente, seja via gasodutos, principalmente da Bolívia, seja via GNL.

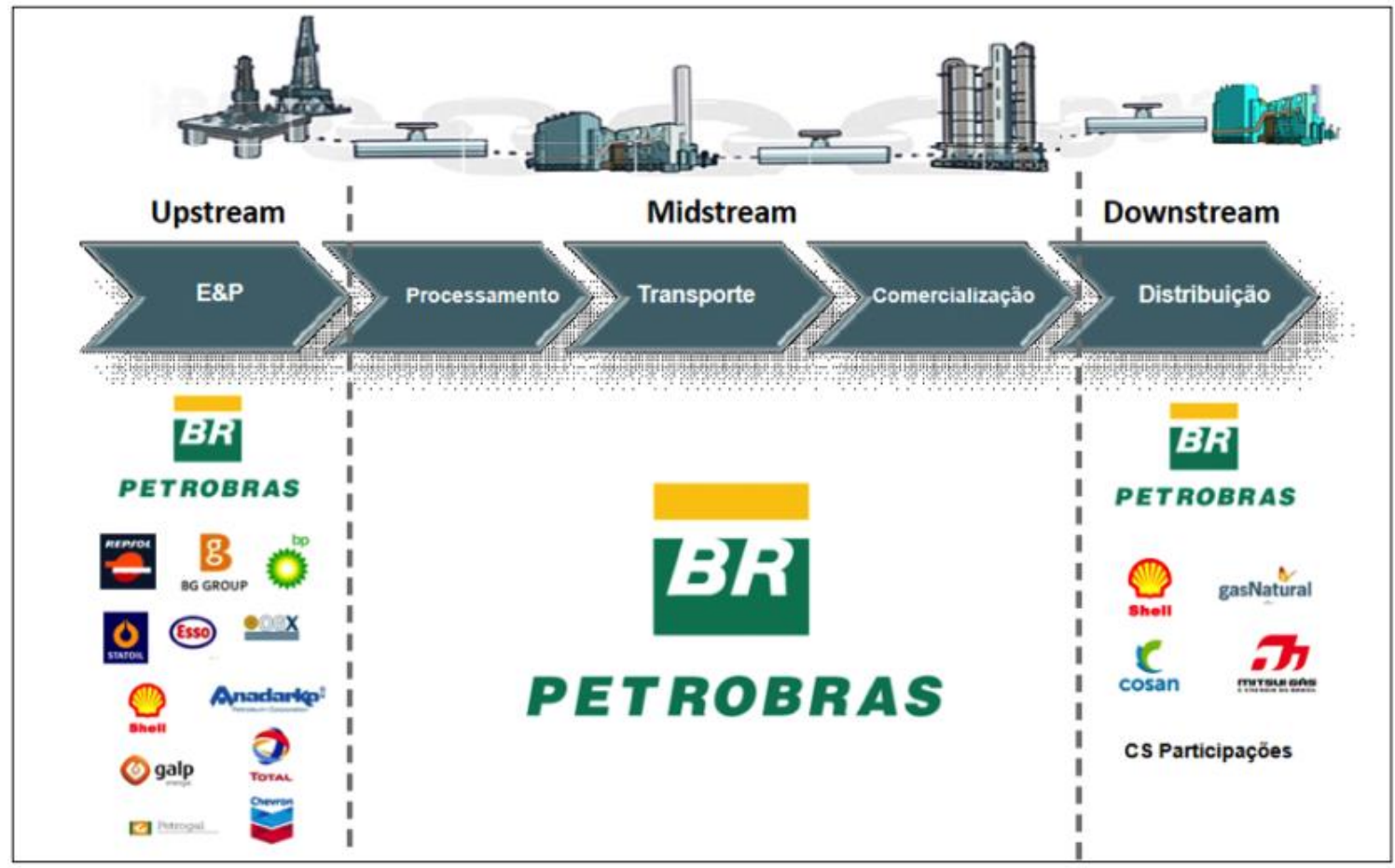

Figura 2. Participação das Empresas em cada Segmento

Fonte: De acordo com a CNI "Qualquer parte desta obra poderá ser reproduzida, desde que citada a fonte". Confederação Nacional da Indústria. (2016). Reestruturação do setor de gás natural: Uma agenda regulatória (p. 91). Brasília: Autor. Recuperado de http://www.fgv.br/fgvenergia/reestruturacao_do_setor_de_gas_natural/files/assets/common/downloads/Reestruturacao_do_Setor_de _Gas_Natural.pdf

Baseado no que já fora exposto, existe a demanda do produto e existe a possibilidade do aumento do consumo se a infraestrutura e rede de gasoduto crescerem, se houver substituição gradual na matriz energética do país favorecendo o consumo do gás natural, se houver maior necessidade dos consumidores, e se for aprovado o projeto de lei que é conhecido como Gás para Crescer que dispõe sobre diversas medidas para fomentar a Indústria de Gás Natural e irá alterar a Lei 11.909, de 4 de março de 2009, conhecida como a Lei do Gás.

Após esse preâmbulo, o enfoque é a SC aplicada a esse panorama. Na Figura, uma demonstração da quantidade de contratos que são necessários para atender a cadeia de fornecimento de gás natural. Essa é uma tentativa para demonstrar os direitos e as obrigações contratuais, evidenciando os mecanismos utilizados para regular as relações interorganizacionais. 
Antes de 2007, a comercialização de gás natural no Brasil dividia-se entre contratos Firmes e Flexíveis, com a Petrobras. Os contratos Firmes eram caracterizados por cláusulas do tipo Take-orPay e Ship-or-Pay, que estabeleciam um compromisso de pagamento por quantidades mínimas contratadas por parte do comprador, independentemente de utilizá-lo ou não. A partir de 2007, a Petrobras, como detentora do monopólio no segmento de midstream, flexibilizou os contratos diante das novas necessidades no mercado e passou a ofertar contratos mais adequados ao perfil de produção e de demanda dos consumidores (ANP, 2011). Para tal, foram criados os modelos Firme Flexível e Preferencial, enquanto outros foram modificados, conforme a seguir:

- Firme inflexível

- Firme flexível

- Interruptível

- Preferencial

- Take-or-Pay

- Ship-or-Pay

Portanto, uma vez assinado o contrato, ele se torna válido, padronizado, mas passível de flexibilidade desde que haja comum acordo entre os membros participantes (Filippi \& Wright, 2018). Portanto, são bons candidatos para a aplicação da SC, pois diante desse cenário e complexidade de termos e condições contratuais, a SC surge como uma alternativa para automação das obrigações estabelecidas no contrato de comercialização do gás natural no Brasil.

A SC é um protocolo de transação computadorizado que executa os termos de um contrato (Szabo, 1994). A SC é baseado na tecnologia chamada Blockchain que, por sua vez, é uma tecnologia de registro de informação, que pode ser em uma rede descentralizada pública ou em uma rede descentralizada privada, isto é, rede aberta apenas entre os agentes escolhidos, mas que possibilita o conceito de ponto-a-ponto, sem utilização de intermediários, como bancos e departamentos inteiros dentro das organizações criadas para averiguar e checar todos os pontos durante a comercialização do gás natural (Swan, 2015).

Além disso, a Blockchain propicia um sistema inviolável para se registrar as ações na SC e que possibilita sincronizar as ações estabelecidas e desenvolvidas dentro de um SC como compra, venda, confirmação de entrega, conversão de moedas, reajustes, emissão de documentos digitais, registro contábil em um livro caixa digital, compartilhamento de informação com os participantes da rede e pagamentos de impostos e direitos, todos em tempo real, sem a possibilidade de erros de cálculos, sem o risco de atraso de pagamento ou não cumprimento de algum termo contratual estabelecido dentro do SC (Swan, 2015). Assim, a SC pode tornar o processo mais previsível ou torná-lo mais eficiente traduzindo-o em mais rápido, menos burocrático e até pode diminuir o custo total das organizações, pois é possível prever a possibilidade de redução na quantidade de departamentos que são necessários atualmente para administrar as tarefas, os termos e condições na comercialização do gás natural nas organizações, fora a possível redução dos custos administrativos existentes no cenário atual (Szabo, 1997).

Além do exposto, também pode reduzir os riscos advindos de erros humanos se a SC puder substituir as ações feitas pelos humanos e permitir um processo interorganizacional automatizado, visível e aberto a todos os participantes, seguro por criptografia e comum a todos (Buterin, 2014).

Do ponto de vista da linguagem de programação, a SC pode ser escrito em várias linguagens e uma delas é a Ethereum (Buterin, 2014). Contudo, já existem empresas que desenvolvem plataformas exclusivas e privadas para SC; específicos e customizados para cada condição e situação, conforme Hyperledger (2016). E podem ser configurados e desenvolvidos com outras linguagens como Python, $\mathrm{C}++$, Java e outras. 
A proposta é automatizar, sincronizar e registrar todas as ações presentes no modelo apresentado na Figura através da SC, graças à tecnologia Blockchain. Seria a automação da gestão do contrato. A linguagem de programação pode ser escrita e desenvolvida de forma customizada para atender a todos os interesses, sem esquecer a parte jurídica. Isso resultaria em um novo modelo de negócio (Yermack, 2017).

\section{Blockchain}

A Blockchain, Smart Contracts e bitcoins são terminologias e conceitos recentes e são comumente confundidos. Esta pesquisa foi feita entre abril de 2017 até janeiro de 2018, na base de dados do ProQuest, Google Scholar, Elsevier, ScienceDirect e Emerald Insight, e constatou que há falta de publicações acadêmicas publicadas em revistas de qualidade A1 e A2, utilizando a base de dados Sucupira/Qualis, para o idioma português sobre esses conceitos no momento que este artigo foi elaborado. As palavras chave na busca foram Blockchain, smart contract e contratos inteligentes, separados e em combinação, usando and e or, sem excluir os livros com intuito exclusivo de mapear os grupos de características do SC, que são apresentados de forma gradativa nas próximas seções. Para colaborar nessa averiguação, Alharby e Moorsel (2017), elaboraram um estudo de mapeamento sistemático sobre tópicos atuais de pesquisa em SC, utilizando os passos descritos na Figura 3. Segundo Petersen, Feldt, Mujtaba e Mattson (2008 como citado em Alharby \& Moorsel, 2017), esse processo para o mapeamento sistemático já foi utilizado com sucesso para procurar documentos relevantes em bases de dados científicas. Os mesmos autores concluem que há falta de qualidade na pesquisa sobre SC e falta de pesquisa sobre aplicação real de SC, além da falta de artigos em português.

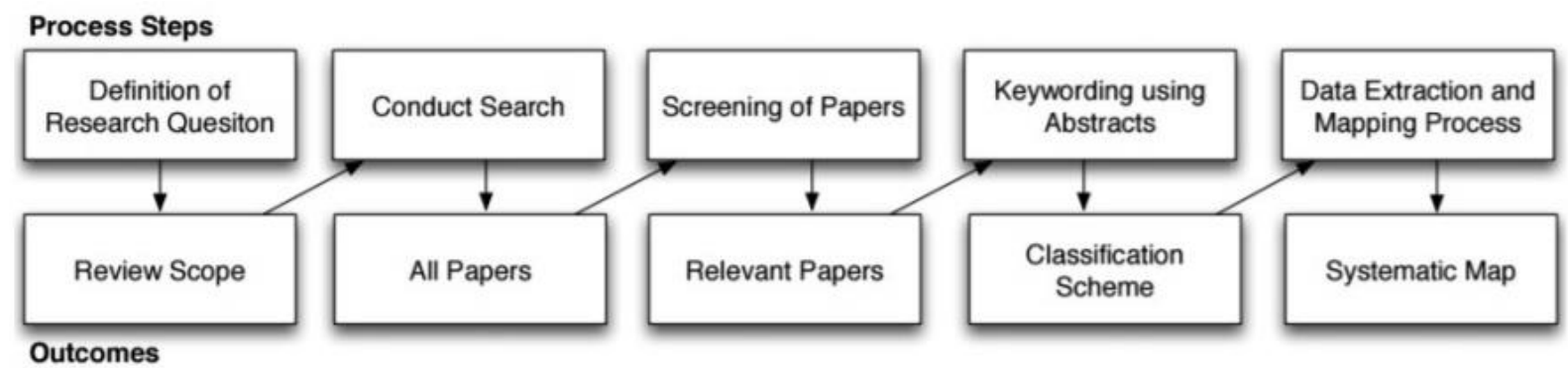

Figura 3. Etapas do Estudo de Mapeamento Sistemático sobre SC Utilizado por Alharby e Moorsel (2017)

Fonte: Petersen, Feldt, Mujtaba and Mattsson (2008 como citado em Alharby, M., \& Moorsel, A. van (2017). A systematic mapping study on current research topics in smart contracts (pp. 2-10). International Journal of Computer Science \& Information Technology, 9(5), 151-164. https://doi.org/10.5121/ijcsit.2017.9511)

Não há como começar a descrever a Blockchain nem SC sem antes mencionar moedas digitais, como o bitcoin (Swan, 2015). A tentativa de conceituar a Bitcoin neste artigo foi feita após análise da literatura emergente sobre criptomoedas que até agora vem da ciência da computação, principalmente. Foi através de artigos e livros em inglês, tendo como exemplo, mas não esgotando as fontes, os seguintes autores: Brière, Oosterlinck e Szfarz (2015), Böhme, Christin, Edelman e Moore (2015), Christidis e Devetsikiotis (2016), Nakamoto (2008) e Swan (2015). O bitcoin é uma moeda digital descentralizada utilizada para pagamentos virtuais utilizando técnicas de criptografia, por isso, também denominada como criptomoeda. Utiliza o conceito de ponto-a-ponto, sem utilização de intermediários, como bancos e empresas. Cabe ressaltar que essa pesquisa não tem a pretensão de compreender as diversas características e aplicações do bitcoin e outras criptomoedas, porém, a tecnologia por trás do bitcoin para satisfazer a abordagem da SC.

Em 1991, uma cadeia de blocos criptograficamente segura foi apresentada por Haber e Stornetta (1990), mas foi apenas em 2008 que a primeira cadeia de blocos foi conceitualizada por Nakamoto 
(2008). Foi implementado e popularizado através da moeda criptografada conhecida como bitcoin (Swan, 2015). Satoshi Nakamoto é um pseudônimo. Até hoje não se sabe quem é realmente a pessoa (ou pessoas) por trás do codinome Nakamoto (Swan, 2015). Isso faz pouca diferença para a pesquisa. Atualmente, o código aberto do bitcoin tem sido reescrito por um grupo de desenvolvedores com identidades conhecidas (Swan, 2015).

Conforme Christidis e Devetsikiotis (2016), a Blockchain é uma tecnologia que mantém um banco de dados distribuído, ou digital ledger, ou livro contábil, para manter um registro de transações de todos os bitcoins já gerados, sempre revelando o endereço de onde saem e para onde foram, ou para onde estão indo. De uma forma a qual todos que estão conectados na rede podem ver, ou seja, infere transparência e acessibilidade. Esta rede é, na sua essência, uma cadeia de computadores na forma distribuída. Quando uma nova transação, ou uma correção de transação, é efetuada por um usuário, temporariamente essa transação espera para ser adicionada em algum bloco, e fica em uma estrutura chamada de pool. Automaticamente, os computadores da rede, ou nós, competem entre si para ver quem consegue encontrar um bloco válido dentro do pool. O primeiro computador que encontra um bloco válido avisa os demais para que se faça a checagem. A informação é recebida e grande parte dos nós, participantes, computadores ou mineiros, da rede da Blockchain deve executar alguns algoritmos e, essencialmente, avaliar e verificar o histórico individual do bloco da Blockchain. A execução desses algoritmos é realizada durante um intervalo de tempo para que haja a concordância de que o histórico e a assinatura são válidos. Corroborando, Böhme et al. (2015) explica que apenas quando considerado válido é que a nova transação será aceita no registro e um novo bloco será adicionado à cadeia de transações com horário definido ou time-stamping. Este processo de registro após a verificação é chamado de mineração. $\mathrm{O}$ chamado nó de mineração, ou mineiro, envia esse bloco de volta à rede e a esse processo foi denominado como prova de trabalho ou em inglês, Proof of Work (PoW).

Ainda segundo Christidis e Devetsikiotis (2016), o papel dos nós, participantes, computadores ou mineiros, é de verificação. O nós verificam se o bloco sugerido contém transações válidas e verificam se aqueles usuários são donos daquela informação; protocolo chamado Proof of Stake (PoS). Além do mais, verificam se as referências, via função hash, estão corretamente atreladas ao bloco anterior em sua cadeia. E se esse é o caso, eles adicionam o bloco à sua cadeia, confirmam as transações e atualizam a cadeia. Caso a maior parte dos nós, participantes, computadores ou mineiros, não reconheça a adição ou modificação da entrada de registro, tal entrada é negada e não é adicionada à cadeia, conforme Böhme et al. (2015) explica. Isso marca o fim de uma rodada de verificação ou transação. Observe que este é um processo de repetição. Então, esse modelo de consenso distribuído é o que permite que a Blockchain funcione como um registro distribuído sem a necessidade de uma autoridade central para certificar quais transações são válidas e quais não são.

Para conceituar a função hash citada anteriormente, Stallings (2005) aborda que é uma função que mapeia um bloco ou mensagem de dados de comprimento variável em um valor de comprimento fixo chamado código hash. A função é projetada de tal forma que, quando protegida, fornece um autenticador para os dados ou mensagem. Para explicar, a função hash cria uma impressão digital ou resumo da mensagem para o bloco. Caso haja uma tentativa de modificação da entrada desse resumo da mensagem, que é um código alfanumérico, a função hash busca o mesmo resumo da mensagem em blocos anteriores e verifica se entrada foi alterada. No entanto, se elas combinarem, é sinal que o código alfanumérico é legítimo, portanto, a modificação da entrada é validada. E esse também é um processo de repetição. Resumindo, o valor hash do bloco anterior é usado para calcular o valor hash do bloco atual, criando esse link entre os blocos. Um outro ponto importante é sobre a irreversibilidade e segurança nessa transação que é feita e validada, característica marcante na Blockchain. Não existe como o remetente acessar novamente todos os dados que seriam necessários para reconstruir, copiar e transmitir aquele mesmo registro ou criptomoeda duas ou mais vezes, conforme elucida Swan (2015). As informações de uma transação são sempre únicas e irreversíveis no tempo e no espaço e essa característica do sistema é assegurada pelo uso avançado da criptografia.

O conceito de criptografia está associado à segurança de informação. Conforme Stallings (2005), criptografia permite a transformação reversível da informação de forma a torná-la ininteligível 
a terceiros. Utiliza-se para tal, algoritmos determinados e uma chave secreta para, a partir de um conjunto de dados não criptografados, produzir uma sequência de dados criptografados.

Segundo Yermack (2017), os mercados emergentes podem estar entre os primeiros a ver a tecnologia Blockchain integrada em suas bolsas de valores e mercados de capitais em grande escala. A previsão do início de adoção nos países em desenvolvimento, segundo ele, depende da convergência de três forças: inadequação de sistemas existentes de manutenção de registros, desconfiança de reguladores de mercado corruptos e ineficazes e alta penetração de tecnologia da informação, como Smartphones. Como exemplo, o autor menciona o rápido crescimento de sistemas de pagamento móvel, como M-Pesa e BitPesa no Quênia, e o recente interesse dos governos de Honduras e da República da Geórgia de mudar seus registros de propriedade, efetuados em cartórios, para Blockchain. Isso fornece uma visão de que as economias emergentes podem adotar essa nova tecnologia em substituição aos antigos processos. E apoiado nesses exemplos, conjectura-se que o Brasil pode utilizar da mesma tecnologia.

A luz da governança, a Blockchain pode contribuir para a transparência de informações dentro do governo brasileiro e para a sociedade por meio de aplicação de tecnologia de informação, também intitulada como e-governança (Guimarães \& Medeiros, 2005). Como todas as informações transacionadas dentro da Blockchain ficam registradas e não podem ser removidas, conforme explica Swan (2015), o governo brasileiro pode utilizá-la como instrumento, cooperando em desenvolver a SC com as organizações de modo que possa facilitar sua aplicação e talvez mudar a inter-relação entre governo e empresas (Ølnes, Ubacht, \& Janssen, 2017).

Após a explicação dos conceitos básicos citados e da sua utilização, o enfoco é aplicar o conceito da Blockchain em SC.

\section{Smart Contracts}

Nicholas Szabo introduziu esse conceito em 1994 e definiu um SC como um protocolo de transação computadorizado que executa os termos de um contrato. Uma forma tradicional de conceituar contrato, conforme cita Swan (2015), seria um acordo entre duas ou mais partes para fazer ou não fazer algo, em troca de outra coisa. Cada parte deve confiar na outra parte para cumprir seu lado da obrigação. Já na literatura brasileira, segundo Roque (2003), o contrato é o acordo de duas ou mais pessoas para estabelecer, regular ou terminar um vínculo jurídico. Os mecanismos de governança de confiança na competência e contrato formal fornecem condições de facilitação única para o aprendizado interorganizacional. Deste modo, o SC possui o mesmo tipo de acordo para cumprir ou não, mas elimina a necessidade de um tipo de verificador entre as partes. Ainda segundo Swan (2015), isso ocorre porque um SC é definido pelo código e executado, ou forçado, pelo código, automaticamente sem astúcia ou manipulação. Ou seja, o funcionamento do SC segue a lógica se X, então Y contida no código pelo qual o contrato será regido. Desse modo, o SC se torna autoexecutável na medida em que o adimplemento da condição desencadeia o resultado.

O Smart Contract, SC, é criado dentro de uma plataforma de aplicações digital. A mais conhecida é a plataforma digital descentralizada criada por Buterin (2014) e Wood (2014) chamada Ethereum. Ethereum, segundo seus criadores, é como um computador mundial que se aproxima de uma máquina virtual, denominada como Ethereum Virtual Machine (EVM), com uma linguagem de computação completa, também conhecida como linguagem de Turing, capaz de resolver diversos problemas usando linguagem computacional de script universal conhecida como solidity, contudo passível de futuro desenvolvimento e atualizações. Ainda segundo Buterin (2014), Ethereum é utilizada para literalmente construir quaisquer atributos matematicamente descritíveis através do mecanismo de contratos. Referida linguagem permite aos desenvolvedores de todas partes do mundo escrever seus próprios contratos, distribuindo-os na rede descentralizada do Ethereum. 
Conforme Buterin (2014), essa plataforma ainda conta com uma criptomoeda assim como o bitcoin. Para esclarecer, primeiro temos o Ethereum, como sistema de pagamento. Em segundo, tem o Ether como moeda do sistema ou como Buterin (2014) prefere denominá-lo, de criptocombustível já que vai manter os contratos ativos do Ethereum. Pois, uma vez que, para ser mantido na rede distribuída, o contrato precisa de fundos para adimplir o custo de manutenção da rede. Esta combinação Ethereum e Ether permite a criação de instruções computacionais programáticas complexas - usualmente conhecidas como SC e aplicações descentralizadas, conforme o mesmo autor explica.

Segundo Szabo (1997), o SC reduz os custos computacionais impostos por terceiros. Ele explica que as fases contratuais de pesquisa, negociação, compromisso, desempenho e adjudicação constituem o domínio dos SC. Os SC utilizam protocolos e interfaces de usuário para facilitar todas as etapas do processo de contratação. Isso fornece novas maneiras de formalizar e proteger as relações digitais que são muito mais funcionais do que a forma tradicional baseada em papéis inanimados.

Ainda conforme Szabo (1997), o intercâmbio eletrônico de dados (IED), confere essa agilidade nos SC. Ele permite a comunicação computador-a-computador de transações comerciais padronizadas entre organizações, em um formato padrão que permite ao receptor realizar a transação pretendida. Ele torna as formas comerciais estáticas tradicionais no ciberespaço e mantém a dependência dos controles tradicionais. Além das verificações simples de criptografia e integridade, o IED não aproveita os algoritmos e protocolos para adicionar segurança e inteligência aos relacionamentos comerciais. Ele permite uma execução mais rápida dos procedimentos tradicionais de negociação e monitoramento de desempenho, conforme corrobora Buterin (2014) e Wood (2014).

Para estabelecer uma semelhança aos fatos mais comuns, se o bitcoin tem o poder de substituir os cartões de crédito e empresas de remessas de dinheiro, o Ethereum tem o poder de substituir entidades que dão suporte aos contratos como são atualmente, ou seja, tem o poder de substituir cartórios, porém com a garantia da criptografia tornando impossível clonar, duplicar ou falsificar um contrato digital dessa modalidade.

Cabe salientar que existe o risco jurídico da implementação de tal plataforma no Brasil. Nesse momento, já existe discussão e proposta de solução para tal risco como a adoção de mecanismos denominado como Judge ou J (Buterin, 2014) em aplicações baseadas em Blockchain como forma de torná-las observantes aos direitos contratuais citados no código civil (Lei n. 10.406, 2002) e consumerista brasileiros. Assim como utilização de outras linguagens de script dentro da Blockchain que visam atender diversas formas de contratos e regulações como o MULTISIG e o CHECKMULTISIG que, segundo Narayanan, Bonneau, Felten, Miller e Goldfeder (2016), podem ser utilizados para conferir características semelhantes a contratos de caução, nos quais há a presença de um comprador, vendedor e um fiel depositário. $\mathrm{O}$ fiel depositário confirma o pagamento ao vendedor no momento em que o comprador acusa o recebimento do produto, ou serviço, contratado e ainda pode servir como intermediador entre as partes, se assim estiver no contrato. Outro aspecto relevante é sobre a possibilidade de mudança de algum termo ou condição que os atores acordam em modificar. Se há o consenso entre as partes e a possibilidade de transformar a ação desejada em linguagem de programação para sua execução, então o SC atende ao interesse, conforme explica Ogilvie (1993). Para corroborar, conforme Christopher (2016), a transparência e o compartilhamento da informação em um empreendimento composto por organizações são de grande importância, assim como a colaboração dos principais envolvidos na elaboração do plano, nesse caso, a proposta de utilização e criação do SC.

O SC pode melhorar mais um aspecto na elaboração contratual: a possibilidade de diferente interpretação da linguagem natural. Conforme explica Ogilvie (1993), certas palavras têm múltiplos significados e interpretações. E, em muitos casos, a linguagem ambígua pode tornar mais fácil para as partes entrar em um acordo contratual, criando flexibilidade em termos de desempenho contratual. No entanto, a ambiguidade ou a redação inadequada também pode ser usada por uma das partes para lutar contra condições contratuais que as partes não querem mais honrar. O SC fornece uma possível solução para essa dificuldade, pois se as partes envolvidas no processo de elaboração do contrato 
querem certeza, então podem usar o SC para garantir que uma condição contratual seja executada, obrigando as partes a permanecerem vinculadas às suas respectivas obrigações.

\section{A Predição do Uso do Smart Contract}

Segundo os autores Maisel e Cokins (2014), o objetivo principal de uma análise de predição nos negócios é identificar como o futuro pode parecer e quais as ações subsequentes precisam ser tomadas. É um processo contínuo para cultivar a tomada de decisão gerencial e operacional que pode afetar os resultados da organização, seja financeiro ou operacional e. assim, facilitar a execução da estratégia. Baseado nessa proposição de predição para tomada de decisão, a abordagem de previsão da utilização do SC foi feita através da tentativa de compreensão de seus atributos inseridos na dinâmica de contratos do mercado de gás natural.

Conforme Swan (2015), corroborado por Ølnes, Ubacht e Janssen (2017), é possível identificar as características dessa tecnologia. São elas: (a) Transparência, pois, é possível ter a visualização de qualquer transação. (b) Descentralizado, porque não há necessidade de um órgão intermediário que aprove a transação ou que determine certos regulamentos de contrato e, portanto, reduz o custo final da transação comercial e aumenta a velocidade das ações. (c) Seguro, já que o banco de dados é imutável, ou seja, consiste em um registro que não pode ser alterado, revisado ou adulterado, nem mesmo para aqueles que operam o banco de dados. Implica em (d) confiança, pois na validação de uma transação requer que outros computadores de outros participantes da plataforma entrem em um consenso para possibilitar que essa transação ocorra. E, por último, (e) automatizado, tornando o processo mais ágil e previsível. Afinal, o software foi desenvolvido para que não haja duplicidade de informação ou que haja informação conflituosa, sendo assim, transações que não respeitem essa regra não são registradas dentro da Blockchain. Diante do exposto, encontra-se na Tabela 1 o resumo das variáveis estudadas neste artigo para o SC e que foram respectivamente abordados e analisados pelos autores identificados durante o estudo de mapeamento sistemático sobre tópicos atuais de pesquisa em SC até janeiro de 2018.

Tabela 1

Resumo dos Atributos Escolhidos para Estudo, com Corroboração dos Autores Pesquisados

\begin{tabular}{lcccc} 
Autores & Transparência & Segurança & Automatização & Jurídico \\
\hline Böhme et al. (2015) & & $\mathrm{x}$ & & \\
\hline Buterin (2014) & $\mathrm{x}$ & $\mathrm{x}$ & $\mathrm{x}$ & $\mathrm{x}$ \\
\hline Christidis e Devetsikiotis (2016) & $\mathrm{x}$ & $\mathrm{x}$ & $\mathrm{x}$ & \\
\hline Christopher (2016) & $\mathrm{x}$ & & & $\mathrm{x}$ \\
\hline Filippi e Wright (2018). & $\mathrm{x}$ & $\mathrm{x}$ & $\mathrm{x}$ & \\
\hline Nakamoto (2008) & $\mathrm{x}$ & & $\mathrm{x}$ & $\mathrm{x}$ \\
\hline Ogilvie (1993) & $\mathrm{x}$ & $\mathrm{x}$ & $\mathrm{x}$ & $\mathrm{x}$ \\
\hline Ølnes et al. (2017) & $\mathrm{x}$ & $\mathrm{x}$ & $\mathrm{x}$ & $\mathrm{x}$ \\
\hline Swan (2015) & $\mathrm{x}$ & $\mathrm{x}$ & $\mathrm{x}$ & $\mathrm{x}$ \\
\hline Szabo (1997) & $\mathrm{x}$ & $\mathrm{x}$ & $\mathrm{x}$ & $\mathrm{x}$ \\
\hline Wood (2014) & $\mathrm{x}$ & $\mathrm{x}$ & $\mathrm{x}$ & $\mathrm{x}$ \\
\hline Yermack (2017) & & & & \\
\hline
\end{tabular}

Nota. Fonte: Elaborada pelo autor. 
Este artigo propõe uma abordagem exploratória para melhor entendimento dos atributos do SC realizada através da pesquisa documental para identificar os principais grupos de atributos do SC (Creswell, 2007). É considerada qualitativa, pois trata de aspectos subjetivos de um determinado problema que é a classificação dos principais atributos do SC (Creswell, 2007) e não há estudos anteriores com aplicação do SC ao mercado de gás brasileiro. A técnica utilizada para coleta de dados sobre os atributos do SC foi a pesquisa documental, respeitando o estudo e a sistemática realizada por Alharby e Moorsel (2017), completados por questionário realizado e recebido em agosto de 2017, com especialistas na área de comercialização de gás natural. O tipo de questionário foi o semiestruturado, com perguntas abertas e fechadas visando registrar e compreender a perspectiva de automação da gestão de contratos, os principais desafios e a percepção da importância de cada atributo associado ao SC.

O questionário foi elaborado após a leitura dos termos e condições gerais de serviço prestado por Transportadora Associada de Gás S.A. e leitura do modelo dos termos e condições gerais de serviço de transporte firme de gás natural prestado por Transportadora Brasileira Gasoduto BolíviaBrasil S.A. As cópias foram obtidas através do portal de acesso à informação do Governo Federal e respondidas pela Ouvidoria-Geral da Petrobras em abril 2017 para o público (http://transparencia.petrobras.com.br/servico-informacao-cidadao - recuperado em 14 abril 2017). O objetivo foi identificar os pontos comuns nos termos e condições aplicados à comercialização do gás natural e que podem ser automatizados através do SC. Como são termos e condições gerais de serviço, foi possível vislumbrar o SC sendo aplicado a diversos pontos como arredondamentos dos números para pagamentos e unidades. Ou reajuste de preço do produto comercializado, ou ainda, a emissão de documentos padronizados para agentes reguladores e governos. Através do Instituto Brasileiro de Petróleo, Gás e Biocombustíveis e seu comitê relacionado ao gás natural, em junho de 2017, foram enviados 20 questionários para especialistas que trabalharam em diferentes empresas no Brasil na comercialização do gás natural.

Além dessa ação, foram enviados mais 20 formulários para diferentes empresas que comercializam gás natural nos EUA, Noruega e Reino Unido. Os contatos nesses países e nessas outras empresas, com essas pessoas, foram obtidos através da rede de relacionamento entre o autor e alguns especialistas no mercado de gás natural. No entanto, apenas dois especialistas responderam ao questionário satisfatoriamente. Um do Brasil e outro dos EUA, que trabalham na mesma empresa e que tem participação expressiva nos principais mercados de comercialização do gás natural nos EUA e Europa. O terceiro especialista, vice-presidente da área de tecnologia também da mesma empresa que os outros dois especialistas trabalham, comunicou-se através do Skype, mas não autorizou a gravação da conversa. Essa conversa aconteceu no momento posterior ao recebimento dos dois questionários respondidos pelos especialistas 1 e 2, em agosto de 2017, e de maneira suficiente. Ele, da Noruega, autorizou apenas a transcrição que esse autor registrou enquanto entrevistava o especialista, portanto, o objetivo foi de averiguar se o terceiro especialista poderia corroborar com os outros dois questionários respondidos, declarando se algumas das características do SC podem satisfazer a transparência, a segurança, a automação e a parte jurídica que o mercado de gás natural necessita e quais seriam os gargalos. E o que se observou através dessa entrevista foi que há consistência nas respostas dos dois especialistas com relação ao potencial dos atributos identificados no SC, conforme Tabela 2 e Tabela 3 que podem ser aplicadas aos contratos de gás natural visando uma otimização do atual processo.

O resumo das respostas mais relevantes obtidas através do questionário respondido por esses dois especialistas são observados na Tabela 2. Conforme Creswell (2007), o pesquisador busca dar significado ao que esses especialistas observam em suas experiências e funções. Há que se notar que houve uma entrevista com o especialista 1 e o especialista 2 para dirimir dúvidas, questionamentos e esclarecer o objetivo dessa pesquisa, de caráter exploratório, na possível utilização do SC para o mercado de gás natural no Brasil e que tais atributos do SC podem auxiliar na automatização do modelo de termos e condições gerais estudado para esse artigo, embutindo os conceitos de segurança, transparência e o respeito à parte jurídica. 
Tabela 2

Resumo das Respostas das Perguntas Semiestruturadas

\begin{tabular}{|c|c|c|}
\hline & Especialista 1 & Especialista 2 \\
\hline \multirow[t]{2}{*}{ Experiência atual ou passada } & \multirow[t]{2}{*}{ Midstream } & Midstream \\
\hline & & Downstream \\
\hline $\begin{array}{l}\text { Posição na empresa atualmente no } \\
\text { mercado de gás natural }\end{array}$ & Gerente & Gerente \\
\hline $\begin{array}{l}\text { Tipos de contrato que já trabalhou ou } \\
\text { trabalha }\end{array}$ & $\begin{array}{l}\text { EFET (Europa), NBP 97(UK), } \\
\text { NAESB (EUA), Contratos longos } \\
\text { maiores que } 20 \text { anos (Europa), } \\
\text { contratos de médio e curto prazos } \\
\text { (Europa) }\end{array}$ & $\begin{array}{l}\text { Contratos para indústria de } \\
\text { mineração (Petrobras e } \\
\text { distribuidora locais), contratos com } \\
\text { produtores de gás em diferentes } \\
\text { países (Peru, Oman e Argentina) }\end{array}$ \\
\hline \multirow[t]{6}{*}{$\begin{array}{l}\text { Principais pontos de disputa/discórdia } \\
\text { durante vigência do contrato de gás } \\
\text { natural }\end{array}$} & $\begin{array}{l}\text { Força maior definição / chamada. } \\
\text { Requisitos de crédito incluindo } \\
\text { tempo de pagamento. }\end{array}$ & $\begin{array}{l}\text { Indenização por danos devido à } \\
\text { falta de suprimento e/ou } \\
\text { especificação. }\end{array}$ \\
\hline & Flexibilidade de volume. & \multirow{2}{*}{$\begin{array}{l}\text { Medição, atualização de preço, } \\
\text { apuração de take or pay \& } \\
\text { cláusulas de make up. }\end{array}$} \\
\hline & \multirow{3}{*}{$\begin{array}{l}\text { Preço incluindo cláusulas de } \\
\text { revisão de preço em contratos de } \\
\text { longo prazo. } \\
\text { Impacto da mudança de } \\
\text { regulamentação nas estruturas de } \\
\text { contrato médio e longo prazo. }\end{array}$} & \\
\hline & & Itens não previstos no contrato. \\
\hline & & $\begin{array}{l}\text { Definição de responsabilidades } \\
\text { quando o contrato envolve } \\
\text { implementação de infraestrutura } \\
\text { (UPGN, gasoduto de escoamento } \\
\text { e etc). }\end{array}$ \\
\hline & & $\begin{array}{l}\text { Combustível substituto, medição, } \\
\text { preço e etc. }\end{array}$ \\
\hline
\end{tabular}

\begin{tabular}{lcc}
\hline $\begin{array}{l}\text { Pontos que podem ser automatizados } \\
\text { pelo SC }\end{array}$ & $\begin{array}{l}\text { Especialista 1 } \\
\text { (Sim ou Não) }\end{array}$ & $\begin{array}{l}\text { Especialista 2 } \\
\text { (Sim ou Não) }\end{array}$ \\
\hline
\end{tabular}

\begin{tabular}{lll}
\hline $\begin{array}{l}\text { Automatização da medição nos pontos de } \\
\text { recebimento e entrega }\end{array}$ & S & S \\
\hline Envio de aceitação de gás desconforme & N & S \\
\hline $\begin{array}{l}\text { Aceitação de pagamentos entre as partes } \\
\text { envolvidas }\end{array}$ & N & S
\end{tabular}

Emissão de documentos, informes e S S relatórios padronizados, dentro do prazo estabelecido entre as partes

\begin{tabular}{lll}
\hline Cálculo automático para o gás não contado & $\mathrm{S}$ & $\mathrm{S}$ \\
\hline $\begin{array}{l}\text { Cálculo automático para o gás necessário } \\
\text { ao comissionamentos e testes na tubulação }\end{array}$ & $\mathrm{N}$ & $\mathrm{S}$ \\
\hline Reajuste do preço & $\mathrm{S}$ & $\mathrm{S}$ \\
\hline $\begin{array}{l}\text { Cálculo e aplicação de taxa de câmbio } \\
\text { acordada entre as partes (se aplicável) }\end{array}$ & $\mathrm{S}$ & $\mathrm{S}$ \\
\hline $\begin{array}{l}\text { Arredondamentos dos números } \\
\text { (pagamento, unidades etc.) }\end{array}$ & $\mathrm{S}$ & $\mathrm{S}$ \\
\hline $\begin{array}{l}\text { Emissão de documentos padronizados } \\
\text { para agentes reguladores e governo (em } \\
\text { geral) }\end{array}$ & $\mathrm{S}$ & $\mathrm{S}$ \\
\hline
\end{tabular}

Nota. Fonte: Elaborada pelo autor por meio dos questionários respondidos e entrevistas realizadas com os especialistas.

RAC, Rio de Janeiro, v. 22, n. 6, art. 5, pp. 903-921, novembro/dezembro, 2018, http://rac.anpad.org.br 
Os pontos que o especialista 1 mencionou como não sendo adequados à automatização só ficaram claros após a entrevista. Ele mencionou que são pontos que não fazem parte dos termos e condições gerais dos contratos que ele tem experiência, portanto, ele compreende que não são passíveis de automatização, a não ser que haja esgotamento e consenso sobre esses pontos.

Além dessas respostas, também foi solicitado para que os especialistas dessem sua avaliação quanto a importância dos principais atributos identificados conforme Tabela 1, atribuindo valores entre 1 e 4, sendo 1 não é importante, 2 é pouco importante, 3 é importante e 4 é muito importante. $\mathrm{O}$ intuito era averiguar o quão relevantes são os atributos identificados na literatura emergente sobre SC.

Tabela 3

\title{
Resumo dos Graus Dados aos Quatro Atributos Identificados para o SC
}

Marque o grau no campo localizado ao lado das frases

Transparência de processo no contexto de governança corporativa

Especialista 1 Especialista 2

\author{
Seguro do sistema automatizado (ex.: contra invasão de hackers)
}

Automatização (importa- se caso seja pouco automatizado, semiautomatizado, totalmente automatizado)

Confiança jurídica entre todas as partes envolvidas (governo, população, organizações)

Nota. Fonte: Elaborada pelo autor por meio dos questionários respondidos e entrevistas realizadas com os especialistas.

O que podemos captar é que há importância dos quatro atributos para os especialistas sendo a parte jurídica e a segurança com um grau maior de relevância ao abordar contratos de gás natural. Nos comentários adicionais feitos pelos especialistas 1 e 2 durante as entrevistas verbais e sem registro de vídeo, apenas transcrições, realizadas após a entrega dos formulários e, confirmado na parte do questionário na qual foi possível fazer esses comentários por escrito, eles mencionaram de forma similar a importância fundamental da automatização dos processos, com transparência e confiabilidade para que esses contratos sejam executados de forma imparcial. O especialista 2, inclusive, cita a automatização de processos visto na câmara de comercialização da energia elétrica, que administra os contratos do mercado regulado e livre de energia elétrica no Brasil e que pode ser seguida como exemplo para futuros debates para o comércio do gás natural no Brasil.

Apesar de apenas dois questionários terem sido respondidos adequadamente, o que podemos observar é que os principais atributos identificados paraSC podem auxiliar os especialistas a automatizarem os processos como são praticados nos modelos atuais de comercialização do gás natural. Há fundamentos teóricos embasados na pesquisa documental realizada nesse artigo para que o SC possa ser utilizado para conferir agilidade, segurança e transparência aos procedimentos comuns que foram identificados nos questionários. No entanto, para fins de enriquecimento e para maximizar as similaridades e as diferenças de informação, novos questionários deveriam atingir um número maior de especialistas. Tais questionários podem ser elaborados com intuito de focar em determinadas características do contrato de gás natural onde o SC possa ser avaliado como solução possível.

Para aumentar a discussão da dinâmica do uso da tecnologia, ampliar o entendimento e fomentar questionamentos são propostos dois cenários divergentes.

O primeiro cenário seria o otimista com o aceno positivo dos três principais players para implementação da tecnologia. Conseguinte, estruturação do SC para atender aos interesses de todas as partes, simular sua utilização colocando seu código de programação à prova, através de depuração do código e testá-la. Assim como estabelecer de forma comum e em conjunto, a manutenção, a correção e 
a retroalimentação do ciclo de aprimoramento de todo o sistema digital e, assim, possibilitar ações e planos de sustentabilidade do sistema para longo prazo. Não obstante, a capacitação e treinamento dos funcionários em vários níveis dentro de cada organização de forma a potencializar a utilização e aumentar a aceitação na implementação da mesma tecnologia procurando sinergias entre os envolvidos. Com sucesso, elevando a possibilidade de aplicação em mercados semelhantes e em outros setores da economia. Há uma clara expectativa de melhoria no processo ao implementar o SC.

O segundo cenário seria o pessimista com relação à implementação dessa nova tecnologia, com ambiente instável no Governo e o não alinhamento dos interesses entre as partes envolvidas, tornando impossível a construção do SC. Uma outra situação de risco seria o possível ataque cibernético à plataforma digital criada para o SC que poderia levar ao colapso do sistema, em um caso extremo. Outra seria a coexistência dos dois sistemas, SC e o atual, não atingindo, portanto, um dos objetivos que seria a substituição do atual modelo físico. Não esgotando todas as possibilidades, outra situação complexa seria a implementação do SC e, após sua implementação, haver um conflito jurídico questionado por algum dos players no qual a linguagem de programação, dentro da plataforma de aplicação digital escolhida, não possa atender ou resolver o questionamento.

Portanto, a partir dos conceitos de predição nos negócios (Maisel \& Cokins, 2014), a proposta de utilização do SC encontra fundamentos que possibilitam a sua implementação no contexto atual no mercado de gás natural brasileiro, englobando uma análise holística de todos os players desse comércio, isto é, Governo, Consumidor e Organização e suas respectivas necessidades. Conforme mencionado por Mishra, Devaraj e Vaidyanathan (2013), as empresas buscam melhorar seu desempenho empregando novas tecnologias nos seus processos, portanto, a SC poderia desafiar o atual modelo das transações, transformando-o em digital, em forma de linguagem de programação e as obrigações estabelecidas automatizadas. Assim como, se o governo brasileiro colaborar com as organizações e facilitar a adoção da SC nesse mesmo contexto, é possível atingir um novo patamar de transparência desse processo interorganizacional e alcançar maior velocidade de informação, ambos satisfazendo um dos princípios da e-governança (Guimarães \& Medeiros, 2005).

\section{Conclusão}

Nessa quarta revolução industrial é de se esperar que todos os setores da economia sofrerão algum tipo de impacto no modelo e na dinâmica dos negócios. A tecnologia Blockchain faz parte dessa revolução em conjunto com o SC. Portanto, o centro decisor ao avaliar o SC como possível solução para automatizar a gestão do contrato para a comercialização do gás natural, deve analisar a proposta em conjunto com todos os players desse comércio, i.e., Governo, Organização e Consumidor. O desafio é mapear todas as obrigações determinadas no contrato de comercialização do gás natural, conforme Figura, e transformá-las em linguagem de programação, conforme Buterin (2014) e Wood (2014) enfatizam. Os direitos, garantias e obrigações que são pertinentes a cada uma das partes pode ser escrito na linguagem de programação escolhida e de consenso com os principais participantes. Ademais, o fator jurídico deve ser tratado de forma holística com risco do não atendimento de todos os interesses.

O potencial de sucesso de utilização pode ser aumentado se desenvolvido em cooperação entre os principais membros participantes. Ainda, eles devem estabelecer e dividir as responsabilidades no sistema digital proposto. $\mathrm{O}$ acesso a informação gerada pelo SC é primordial para todos os envolvidos pois gera transparência em todo o processo, no contexto de governança corporativa, e consequentemente, confiança. No que tange ao método de pagamento entre as partes, incluindo os impostos, esse deve ser discutido de maneira aprofundada pois, conforme exposto neste artigo de caráter exploratório do tema, pode envolver uma unidade de moeda digital já que as transações são em tempo real e necessitam ser registradas na plataforma digital escolhida para criação do SC, ou dentro da Blockchain. Cabe ressaltar que se uma moeda digital for planejada, esta poderá ser convertida para moeda corrente no país desde que seja estabelecido o prazo para conversão e pagamento, todos 
registrados no SC. A razão de conversão também fica registrada no SC. Assim, todo o fluxo é automatizado e sincronizado, tornando-o mais previsível e menos passível ao erro de origem humana.

Além do exposto, a manutenção, a correção e a retroalimentação do ciclo de aprimoramento de todo o sistema digital, portanto, as ações de sustentabilidade do sistema digital para longo prazo, devem ser constituídas e suas responsabilidades definidas e acordadas entre as partes, incluindo o treinamento e capacitação em diversos níveis em cada organização, com intuito de sinergia e comum entendimento. Com o êxito dessa utilização, é esperado a replicação em mercados semelhantes e em outros setores da economia.

Baseado nos conceitos apresentados e na tentativa de previsão do uso da tecnologia, através da apresentação de dois cenários de aproximação, supõe-se o possível atendimento de todas as partes envolvidas no processo. Porém, é preciso que haja consenso entre as partes envolvidas ao mesmo tempo que o SC contemple todas as cláusulas com direitos, deveres e possível flexibilidade acolhendo as necessidades das organizações. Isso implicará na automação da gestão do contrato para a comercialização do gás natural. Para tal, tais cláusulas de longo prazo devem ser traduzidas para linguagem de programação para entregar o resultado esperado. O SC pode agrupar os interesses do principal agente nacional na área de midstream, da agência reguladora e do governo. Além das organizações que exploram e vendem o gás natural, das atuais e futuras companhias de transporte e das companhias de distribuição do gás natural, conhecidas como concessionárias, CEG, BAHIAGAS, COMGAS e outras e, por fim, o consumidor final, com a vantagem de ser um processo transparente, seguro de fraudes e com velocidade nas transações.

Outro ponto é a questão voltada para a capacitação de recursos humanos e a sociedade (Tapscott $\&$ Tapscott, 2017), mas que foge do propósito de análise desse trabalho.

Sobre o aspecto jurídico entre todas as partes envolvidas, i.e., Governo, Organização e Sociedade, compete advertir a maneira como o papel da lei é percebido nos dias atuais e talvez possa exigir uma mudança nesse contexto durante a adoção de novas regras tecnológicas. Talvez seja preciso repensar os mecanismos usados para regular esse mercado, a fim de imprimir uma forma mais atualizada com o surgimento desse novo conjunto de regras tecnológicas possíveis graças a SC e a possível fluência das relações de mercado.

No momento que este artigo foi escrito, ainda havia falta de exemplos práticos e de artigos científicos de alta qualidade abordando o assunto aqui tratado, caracterizando este artigo como exploratório na abordagem do problema eu se refere a aplicar a SC na automação da gestão de contratos para comercialização do gás natural no Brasil. Para colaborar nessa averiguação, Alharby e Moorsel (2017), elaboraram um estudo de mapeamento sistemático sobre tópicos atuais de pesquisa em SC, utilizando os passos descritos na Figura 3, que o concluíram. Outro limitante foi a quantidade de questionários respondidos por especialistas. Apesar de não haver uma base de dados sobre a quantidade de especialistas em contratos de comercialização de gás natural atuando no Brasil, apenas 2 questionários foram respondidos de forma satisfatória dentre os 40 questionários enviados. No entanto, abre-se espaço para que o modelo de pesquisa e questionário proposto seja aprimorado. Por conseguinte, mesmo dentro de suas limitações, acredita-se que tais limitadores não invalidam o trabalho como um esforço de pesquisa empírica e exploratória. Este artigo alcançou seu objetivo ao abranger a descrição da nova tecnologia, ao aprofundar o conhecimento na explicação de seus atributos, ao fornecer uma melhor compreensão da sua possível utilização no mercado de gás brasileiro, e ao tentar alcançar a lacuna identificada para pesquisas que considerassem o SC em outras aplicações de forma exploratória, além de lançar base para futuros estudos, conforme identificado por Alharby e Moorsel (2017).

Para pesquisas futuras, é recomendado uma análise de caso real com aplicação da SC para a tentativa de refutar ou ajustar a proposta desse artigo. Outra recomendação é a discussão e exaustão deste artigo com artigos de maior qualidade científica nos conceitos aqui apresentados de caráter exploratório ou aprimoramento do modelo com apoio do resultado de mais entrevistas com mais 
especialistas. Nessa linha, pode ser utilizado um outro método de apoio à tomada de decisão para averiguar os atributos da SC.

\section{Referências}

Agência Nacional do Petróleo, Gás Natural e Biocombustíveis (2011). Análise da regulamentação, da estrutura da indústria e da dinâmica de formação dos preços do gás natural no Brasil. p. 44, 2011. Recuperado em 22 de outubro, 2017, de http://www.anp.gov.br/?dw=58828.

Alharby, M., \& Moorsel, A. van (2017). A systematic mapping study on current research topics in smart contracts. International Journal of Computer Science \& Information Technology, 9(5), 151-164. https://doi.org/10.5121/ijcsit.2017.9511

Böhme, R., Christin, N., Edelman, B., \& Moore, T. (2015). Bitcoin: Economics, technology, and governance. Journal of Economic Perspectives, 29(2), 213-38. http://doi.org/10.1257/jep.29.2.213

Brière, M., Oosterlinck, K., \& Szafarz, A. (2015). Virtual currency, tangible return: Portfolio diversification with bitcoin. Journal of Asset Management, 16(6), 365-373. http://doi.org/10.1257/jep.29.2.213

Buterin, V. (2014). A next-generation smart contract and decentralized application platform. $\begin{array}{llllll}\text { Recuperado em } & 7 & \text { de } & \text { novembro, } & \text { 2017, }\end{array}$ https://www.weusecoins.com/assets/pdf/library/Ethereum_white_paper-

a_next_generation_smart_contract_and_decentralized_application_platform-vitalik-buterin.pdf

Carvalhinho, J. C. L., Filho (2003). O valor da flexibilidade em cláusulas take-or-pay de contratos para fornecimento de gás natural industrial (Dissertação de mestrado). Faculdade de Economia, Administração e Contabilidade, Universidade de São Paulo, São Paulo, SP, Brasil. Recuperado de http://www.teses.usp.br/teses/disponiveis/12/12139/tde-01102003232151/en.php

Christidis, K., \& Devetsikiotis, M. (2016). Blockchains and smart contracts for the internet of things. IEEE Access, 4, 2292-2303. http://doi.org/10.1109/ACCESS.2016.2566339

Christopher, M. (2016). Logistics \& supply chain management (5th ed.). Harlow, UK Pearson.

Confederação Nacional da Indústria. (2016). Reestruturação do setor de gás natural: Uma agenda regulatória. Brasília: Autor. Recuperado de http://www.fgv.br/fgvenergia/reestruturacao_do_setor_de_gas_natural/files/assets/common/dow nloads/Reestruturacao_do_Setor_de_Gas_Natural.pdf

Creswell, J. W. (2007). Projeto de pesquisa: Métodos qualitativo, quantitativo e misto (2a ed.). Porto Alegre: Artmed.

Filippi, P. de, \& Wright, A. (2018). Blockchain and the law: The rule of code. Cambridge, Massachusetts: Harvard University Press.

Gomes, I. (2014). Brazil: Country of the future or has its time come for natural gas? [Working Paper NG 88]. The Oxford Institute for Energy Studies. Recuperado de https://ora.ox.ac.uk/objects/ora:10425.

Guimarães, T. A., \& Medeiros, P. H. R. (2005). A relação entre governo eletrônico e governança eletrônica no governo federal brasileiro. Cadernos EBAPE.BR, 3(4), 1-18. Recuperado de 
http://www.scielo.br/pdf/cebape/v3n4/v3n4a04.pdf. 39512005000400004

Haber, S., \& Stornetta, W. S. (1990). How to time-stamp a digital document. Journal of Cryptology, 3(2), 99-111. https://doi.org/10.1007/BF00196791

Hyperledger. (n.d). Hyperledger - Blockchain technologies for business. Recuperado em 22 de outubro, 2017, $\quad$ de https://www.hyperledger.org/wpcontent/uploads/2018/11/Hyperledger_DataSheet_11.18_Digital.pdf

Lei n. 9.478, de 6 de agosto de 1997. (1997). Dispõe sobre a política energética nacional, as atividades relativas ao monopólio do petróleo, institui o Conselho Nacional de Política Energética e a Agência Nacional do Petróleo e dá outras providências. Diário Oficial da República Federativa do Brasil, Poder Legislativo. Brasília, DF.

Lei n. 10.406, de 10 de janeiro de 2002. (2002). Institui o Código Civil. Revisão até 9 de junho, 2016. (9a ed.). (Edições Câmara). Brasília: Câmara dos Deputados. Recuperado em 28 de fevereiro, 2018, de http://livraria.camara.leg.br/codigo-civil.html

Lei $n^{\circ} 11.909$, de 4 de março de 2009. (2009). Dispõe sobre as atividades relativas ao transporte de gás natural, de que trata o art. 177 da Constituição Federal, bem como sobre as atividades de tratamento, processamento, estocagem, liquefação, regaseificação e comercialização de gás natural; altera a Lei no 9.478, de 6 de agosto de 1997; e dá outras providências. Recuperado de http://www2.camara.leg.br/legin/fed/lei/2009/lei-11909-4-marco-2009-586820-norma-pl.html

Maisel, L. S., \& Cokins, G. (2014). Predictive business analytics: Forward looking capabilities to improve business performance. New Jersey, USA: Wiley.

Ministério de Minas e Energia. (2014). Plano nacional de energia, 2050 - Cenário sócio-econômico e demanda de energia. Recuperado em 7 de novembro, 2017, de http://www.mme.gov.br/documents/10584/10745/PNE_2050_workshop_eco_dem_vf.pdf/4203 881d-d70b-4050-910e-d4dab0c6600e

Mishra, A. N., Devaraj, S., \& Vaidyanathan, G. (2013). Capability hierarchy in electronic procurement and procurement process performance: An empirical analysis. Journal of Operations Management, 31(6), 376-390. https://doi.org/10.1016/j.jom.2013.07.011

Nakamoto, S. (2008). Bitcoin: A peer-to-peer electronic cash system. Recuperado em 7 de novembro, 2017, de https://bitcoin.org/bitcoin.pdf

Narayanan, A., Bonneau, J., Felten, E., Miller, A., \& Goldfeder, S. (2016). Bitcoin and cryptocurrency technologies: A comprehensive introduction. New Jersey, USA: Princeton University Press.

Ogilvie, J. W. L. (1993). Defining computer program parts under learned hand's abstractions test in software copyright infringement cases. Michigan Law Review Association, 91(3), 526-570.

Ølnes, S., Ubacht, J., \& Janssen, M. (2017). Blockchain in government: Benefits and implications of distributed ledger technology for information sharing. Government Information Quarterly, 34(3), 355-364. http://doi.org/10.1016/j.giq.2017.09.007

Roque, S. J. (2003). Direito contratual civil-mercantil (2a ed.). São Paulo: Ícone.

Stallings, W. (2005). Cryptography and network security: Principles and practices (4th ed.). New Jersey, USA: Prentice Hall.

Swan, M. (2015). Blockchain: Blueprint for a new economy. California, USA: O'Reilly Media. 
Szabo, N. (1994). Smart contracts. Recuperado em 2 de abril, 2017, de http://www.fon.hum.uva.nl/rob/Courses/InformationInSpeech/CDROM/Literature/LOTwintersc hool2006/szabo.best.vwh.net/smart.contracts.html

Szabo, N. (1997). Formalizing and securing relationships on public networks. First Monday, 2(9). Recuperado em 2 de dezembro, 2017, http://firstmonday.org/ojs/index.php/fm/article/view/548/469

Tapscott, D., \& Tapscott, A. (2017). How Blockchain will change organizations. MIT Sloan Management Review, 58(2), 10-13.

Wood, G. (2014). Ethereum: A secure decentralised generalised transaction ledger. Byzantium Version 2d0661f - 2018-11-08. Recuperado em 7 de novembro, 2017, de https://ethereum.github.io/yellowpaper/paper.pdf

Yermack, D. (2017). Corporate governance and Blockchains. Review of Finance, 21(1), 7-31. https://doi.org/10.1093/rof/rfw074

\section{Autor}

Romulo Benites de Souza Luciano

Av. Pres. Wilson, 118, 20030-020, Centro, Rio de Janeiro, RJ, Brasil.

E-mail: romulo.benites@gmail.com 